\title{
Modulating Effects of Mannose Binding Lectin Genotype on Arterial Stiffness in Children After Kawasaki Disease
}

\author{
YIU-FAI CHEUNG, MARCO H.K. HO, WAI-KEE IP, SUSANNA F.S. FOK, \\ TAK-CHEUNG YUNG, AND YU-LUNG LAU \\ Department of Paediatrics and Adolescent Medicine [Y.-F.C., M.H.K.H., W.K.I., S.F.S.F., T.-C.Y., Y.-L.L.], \\ The University of Hong Kong, Queen Mary Hospital, Hong Kong, China
}

\begin{abstract}
ABST
Systemic arterial stiffness is increased in patients after Ka-
wasaki disease (KD). Recently, associations between mannose-
binding lectin (MBL) gene mutation and coronary complications
in infants with KD and atherosclerosis in adults have been
reported. We tested the hypothesis that $M B L$ genotype modulates
arterial stiffness in children after KD. Seventy-one KD patients
(42 with and 29 without coronary aneurysms), aged $9.5 \pm 3.7$ y,
and 41 age-matched controls were studied. We determined and
compared their blood pressure, brachioradial arterial stiffness as
determined by pulse wave velocity (PWV), fasting total choles-
terol, serum MBL level, and $M B L$ genotype. Additionally, the
modulating effects of different $M B L$ expression genotypes [high
level (HL) versus intermediate or low level (IL/LL)] on arterial
stiffness in different groups were assessed. The $M B L$ genotype
distributions did not differ between patients and controls $(p=$
$0.41)$ or between patients with and without coronary aneurysms
( $p=0.42)$. Patients with IL/LL expression genotypes had sig-
nificantly faster PWV than those with HL expression genotypes
(7.93 $\pm 1.38 \mathrm{~m} / \mathrm{s}$ versus $6.67 \pm 2.28 \mathrm{~m} / \mathrm{s}, p=0.027)$. This
\end{abstract}
genotype-modulating effect is more pronounced in patients without $(\mathrm{HL} 8.86 \pm 0.77 \mathrm{~m} / \mathrm{s}$ versus IL/LL $6.48 \pm 2.32 \mathrm{~m} / \mathrm{s}, p=$ $0.02)$ than those with (HL $7.50 \pm 1.41 \mathrm{~m} / \mathrm{s}$ versus IL/LL $6.80 \pm$ $2.28 \mathrm{~m} / \mathrm{s}, p=0.32$ ) coronary aneurysms. Multiple linear regression analysis identified age $(\beta=0.26, p=0.012)$, being a Kawasaki patient $(\beta=0.22, p=0.015)$, and $M B L \mathrm{IL} / \mathrm{LL}$ genotype subgroup $(\beta=0.20, p=0.03)$ as significant determinants of arterial stiffness in the entire cohort. In conclusion, $M B L$ genotype modulates arterial stiffness, an important cardiovascular risk factor, in children after KD. (Pediatr Res 56: 591-596, 2004)
HL, high level
IL, intermediate-level
KD, Kawasaki disease
LL, low level
MBL, mannose-binding lectin
PWV, pulse wave velocity

$\mathrm{KD}$, a systemic vasculitis with a predilection for Oriental children, is presently the commonest acquired heart disease in children in developed countries (1). Whereas structural (2) and functional $(3,4)$ alterations of coronary arteries as a consequence of coronary arteritis have been well documented, the long-term impact of diffuse vasculitis on systemic arterial function is just beginning to be unveiled (5-7). We (7) and the others $(6,8)$ have shown that an adverse cardiovascular profile, as characterized by proatherogenic alteration of lipid profile and increased arterial stiffness, occurs in patients late after KD.

Received February 11, 2004; accepted April 26, 2004.

Correspondence: Y.-F. Cheung, MBBS, FRCP, MRCPCH, FHKAM (Paediatrics), Department of Paediatrics and Adolescent Medicine, The University of Hong Kong, Queen Mary Hospital, Pokfulam Rd., Hong Kong, China; e-mail: xfcheung@hkucc.hku.hk

Supported by a CRCG Research Grant (Y.-F.C.), Faculty of Medicine, The University of Hong Kong and a Paediatric Departmental Research Grant (M.H.K.H.), Department of Paediatrics and Adolescent Medicine, The University of Hong Kong.

DOI: 10.1203/01.PDR.0000139406.22305.A4
Furthermore, there is evidence to suggest that the increase in systemic arterial stiffness may be related to the severity of inflammation during the acute phase $(7,9)$. In fact, delayed administration of i.v. Ig therapy has been shown to exaggerate the increase in peripheral conduit arterial stiffness in the long term (9). Hence immune modulation by either extrinsic or intrinsic mechanisms during the acute phase of KD may play a role in determining long-term stiffness of the arteries after vasculitic damage.

MBL is a collagenous lectin that binds to mannose and $N$-acetyl glucosamine residues on the surface of many microbial antigens (10). Binding of MBL activates complements independently of antibodies and leads to opsonization of the microorganisms $(11,12)$. Deficiency of $\mathrm{MBL}$ due to point mutations of the $M B L$ gene has been shown to predispose to infectious illnesses in both children (13-15) and adults (16). Recently, associations between $M B L$ gene mutation and development of coronary artery lesions in infants with KD (17) and 
severe atherosclerosis in adults have been reported (18). Indeed, concerns have been raised regarding the possibility of predisposition to premature atherosclerosis in adulthood as a result of systemic arterial dysfunction after $\operatorname{KD}(7,19,20)$.

The serum MBL concentration is genetically determined. Three point mutations associated with low MBL concentration have been identified in codons 52, 54, and 57 of exon 1 in the $M B L$ gene (10). The normal wild-type allele is designated $A$, whereas the variant alleles are designated $O$ (13). In ethnic Chinese, codon 52 mutation occurs at an extremely low frequency (21), whereas codon 57 mutation is probably absent (22). Polymorphisms of the promoter region of the $M B L$ gene have additional effects on serum MBL concentration. In particular is the one at position $-221(Y / X)$, with the $Y$ promoter variants being responsible for a high and $X$ promoter variants for a low $M B L$-expressing activity, respectively (23).

We hypothesize that the $M B L$ genotype modulates arterial stiffness of children with a history of KD. To test this hypothesis, we compared the peripheral conduit arterial stiffness of $\mathrm{KD}$ patients and healthy controls with HL $M B L$ expression genotypes to those with IL or LL $M B L$ expression genotypes.

\section{METHODS}

Subjects. Patients with a history of KD were recruited from the cardiac clinic. Those diagnosed to have KD within 12 mo of the study were excluded to minimize potential confounding influence relating to subacute inflammation (24). From the medical records, the following patient data were retrieved: age at diagnosis of $\mathrm{KD}$, use of i.v. Ig within $10 \mathrm{~d}$ of illness, interval from disease onset to the time of study, and coronary complications. Coronary aneurysms were documented by serial twodimensional echocardiography. Healthy age-matched subjects were recruited as controls. These were healthy children previously discharged from the clinic with a diagnosis of functional heart murmur and their healthy siblings. The institutional ethics committee approved the study and parents of all subjects gave written, informed consent.

The subjects attended for study after an overnight fast. Body weight and height were measured, and body mass index was calculated accordingly. All subjects rested for at least $15 \mathrm{~min}$ before blood pressure and arterial stiffness assessments. Blood pressure in the right arm was measured twice using an automatic oscillometric device (DINAMAP, Critikon Inc., Tampa, FL), with the subjects in the seated position, and the average of the two readings was taken. Assessment of the brachioradial arterial stiffness and blood taking were then performed sequentially, as described below. Venous blood was withdrawn for measurement of fasting total cholesterol, assay of serum MBL concentration, and $M B L$ genotyping.

Measurement of arterial stiffness. Stiffness of the brachioradial arterial segment was assessed by measuring PWV, which is related to the square root of elastic modulus according to the Moens-Korteweg equation (25). Hence, the stiffer the artery, the faster the PWV. A previously validated photoplethysmographic technique (26) was used to measure the transit time required for the pulse to travel from the brachial artery at the elbow to radial artery at the wrist. Briefly, two probes, each containing an infrared emitting diode and a phototransistor, were placed over the right brachial and right radial arteries and secured without compression. As the pulse wave passes along and distends the artery under the probe, more of the infrared signal is absorbed and less reflected. The signals from the two probes were amplified, displayed in real time on the monitor, and sampled by an analog-to-digital converter. The transit time was determined from the time delay between the foot of the corresponding brachial and radial pulse waves. The average of three readings was taken. PWV was calculated by dividing distance between the two points by transit time. Intraobserver variability for measurement of PWV, as reported previously, was $0.08 \pm 0.82 \mathrm{~m} / \mathrm{s}(7)$.

MBL assay and genotyping. Whole blood was collected using potassium-EDTA tubes and frozen at $-70^{\circ} \mathrm{C}$ until DNA extraction, and the serum was separated by centrifugation was similarly stored until MBL assay. Serum MBL concentration was measured by ELISA as described previously (27), which detects mainly fully active oligomerized MBL. DNA was extracted from the blood samples by standard techniques. Genotyping of $M B L$ promoter polymorphisms $(Y / X)$ and codon 54 mutations was performed using two separate cycling reactions of the TaqMan allele-discrimination system (Applied Biosystems, Foster City, CA).

For genotyping of the $Y / X$ promoter polymorphism, genomic DNA was amplified using $1 \mu \mathrm{L}$ of aqueous DNA (approximately $10 \mathrm{ng} / \mu \mathrm{L}$ ) in a final volume of $20 \mu \mathrm{L}$ of $1 \times$ TaqMan Universal PCR Master Mix (Applied Biosystems), $0.9 \mu \mathrm{M}$ of each of 5'-TGG GTT TCC ACT CAT TCT CA-3' and 5'-GAG GGG TTC ATC TGT GCC TA-3' primers (Proligo, Boulder, $\mathrm{CO}$ ), and $0.25 \mu \mathrm{M}$ of each of the following TaqMan probes labeled with a fluorescent dye (Fam or Vic) at the $5^{\prime}$ end and a quencher (Tamra) at the $3^{\prime}$ end (Applied Biosystems): FamTCT CAC TGC CAC GGA AAG CAT GTT-Tamra (allele $Y$ ) and Vic-TCT CAC TGC CAC CGA AAG CAT GTT-Tamra (allele $X$ ). The amplification was carried out at $95^{\circ} \mathrm{C}$ for 10 min, followed by 40 cycles at $60^{\circ} \mathrm{C}$ for $15 \mathrm{~s}$ in $0.2 \mathrm{~mL}$ TaqMan optical tubes using an ABI Prism 7700 Sequence Detector (Applied Biosystems).

For detection of codon 54 mutations, the reactions were carried out in a similar fashion but with 40 cycles at $63^{\circ} \mathrm{C}$ and use of the following primers and TaqMan probes: $5^{\prime}$-CCC TGC AGT GAT TGC CTG TA-3' and 5'-CAG AAC AGC CCA ACA CGT ACC-3'; Fam-ATG GGC GTG ATG GCA CCA AGG-Tamra (allele $A$ ) and Vic-ATG GGC GTG ATG ACA CCA AGG G-Tamra (allele $B$ ). Fluorescence monitoring was performed according to the manufacturer's directions (ABI Prism 7700 Sequence Detector, Applied Biosystems).

Three $M B L$ genotype subgroups were defined, based on the promoter activities and codon 54 mutations: group I comprised $Y A / Y A$ and $Y A / X A$ patients, who have high MBL levels; group II comprised $X A / X A$ and $Y A / O$ patients who have serum MBL levels that are reduced 2- to 4-fold (28); group III included those with $X A / O$ and two defective alleles $O / O$, who have virtually undetectable MBL in the blood (29). The subjects, including patients and controls, were then categorized into those having HL $M B L$ expression genotypes and those with IL or LL $M B L$ expression genotypes. 
Data analysis. Data are presented as mean \pm SD unless otherwise stated. Differences in demographic data and cardiovascular parameters between patients and controls, and those between patients with HL $M B L$ expression genotypes and patients with IL or LL $M B L$ expression genotypes were compared by unpaired $t$ test or Mann-Whitney test where appropriate. Differences in PWV between two subgroups were compared by unpaired $t$ test. Linear regression was performed to assess relation between PWV and age in patients and controls, and the two regression lines were compared using analysis of covariance. Serum MBL concentrations in the three $M B L$ genotype subgroups were compared using Kruskal-Wallis test, with posthoc comparisons by the Dunn's test, whereas distributions of $M B L$ expression genotypes between groups were compared by $\chi^{2}$ or Fisher's exact test. Multiple linear regression was used to identify significant determinants of arterial stiffness. Statistical significance was defined as a $p$ value of $<0.05$. All statistical analyses were performed using SPSS version 10.0 (SPSS, Inc., Chicago, IL).

\section{RESULTS}

Subjects. A total of 112 ethnic Chinese subjects were studied, including 71 children with a history of KD and 41 controls. Of the $71 \mathrm{KD}$ patients, 42 had either persistent or regressed coronary aneurysms, whereas 29 patients had no coronary complications. All but five patients had received i.v. Ig therapy during the acute phase of illness. The patients were studied at $7.7 \pm 3.9 \mathrm{y}$ after $\mathrm{KD}$, all of whom remained asymptomatic and none required coronary artery interventions. The demographic data and cardiovascular risk factors of patients and controls are summarized in Table 1. There were no significant differences in age, sex distribution, body mass index, systemic blood pressure, or fasting total cholesterol between the two groups.

MBL genotypes and serum concentration. The serum MBL concentrations of the three genotype subgroups are shown in Figure 1. As expected, the median serum MBL concentration was significantly greater in the HL expression genotypes group than that in the IL $(p<0.001)$ or LL $(p<0.001)$ expression genotype groups. The $M B L$ genotypic distribution in the controls was similar $(p=0.87)$ to that reported previously in the Chinese population (30) (Table 2). The distributions of $M B L$ genotypes in patients and controls are summarized in Table 3. The $M B L$ genotype distributions did not differ between patients and controls $(p=0.41)$ or between patients with and without coronary aneurysms $(p=0.42)$.

Table 1. Comparisons of demographic data and cardiovascular risk factors between patients and control subjects

\begin{tabular}{lccc}
\hline & Patients $(n=71)$ & Controls $(n=41)$ & $p$ Value \\
\hline Age (y) & $9.5 \pm 3.7$ & $10.5 \pm 3.7$ & 0.17 \\
Sex (male:female) & $48: 23$ & $28: 13$ & 1.00 \\
BMI $\left(\mathrm{kg} / \mathrm{m}^{2}\right)$ & $16.7 \pm 2.8$ & $17.5 \pm 3.2$ & 0.20 \\
Total cholesterol (mmol/L) & $4.4 \pm 0.8$ & $4.2 \pm 0.8$ & 0.29 \\
SBP (mm Hg) & $109 \pm 12$ & $109 \pm 11$ & 0.88 \\
DBP (mm Hg) & $58 \pm 8$ & $58 \pm 8$ & 0.88 \\
\hline
\end{tabular}

BMI, body mass index; DBP, diastolic blood pressure; SBP, systolic blood pressure.

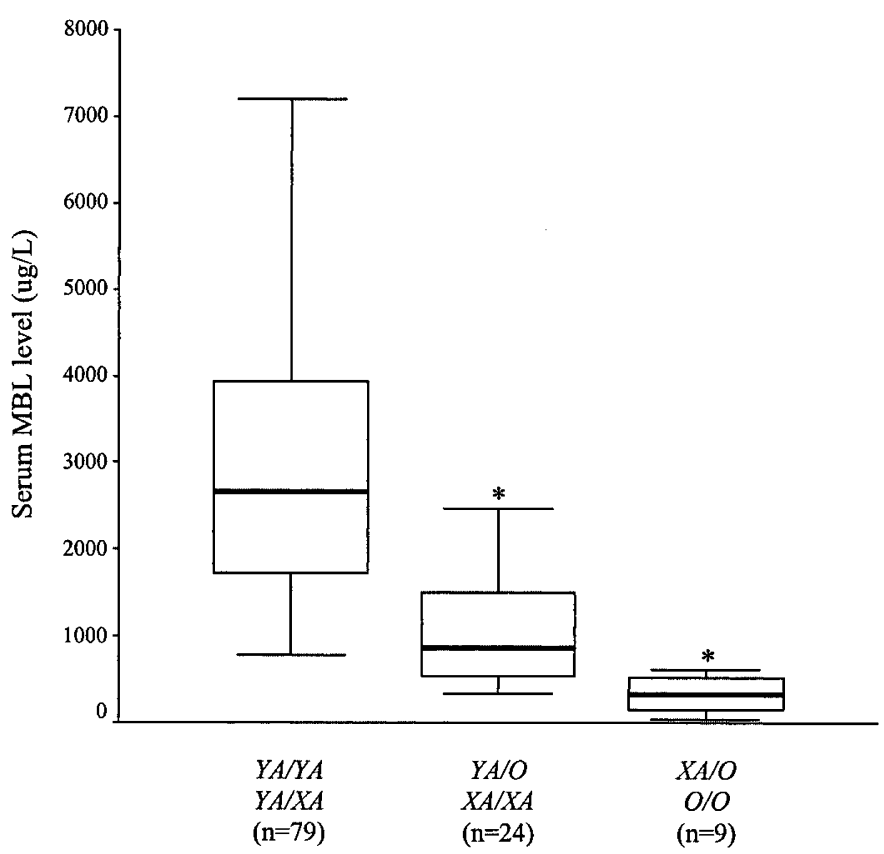

Figure 1. Box plots of serum MBL level in the three $M B L$ genotype subgroups. The line within each box represents the median in each group. $\left({ }^{*} p<0.001 v s \mathrm{HL}\right.$ expression genotypes group).

Modulating effects of MBL genotype on arterial stiffness. The PWV of patients $(7.2 \pm 2.8 \mathrm{~m} / \mathrm{s})$ was significantly higher than that of controls $(6.2 \pm 1.5 \mathrm{~m} / \mathrm{s}, p=0.034)$. Figure 2 shows the linear regression analyses of PWV versus age for the two groups. Although the slopes of the two regression lines were similar $(p=0.48)$, the $y$-intercept of the regression line of patient group was significantly greater than that of control group ( $p=0.007)$, suggesting that, although PWV increased with age in both groups, patients had significantly higher PWV than controls throughout the age range.

To determine potential modulating effects of $M B L$ genotypes on arterial stiffness, the cohort was subcategorized, based on the expected $M B L$ expression, into two groups: HL versus IL/LL (Table 4). The influence of $M B L$ expression genotypes on PWV in patients and controls is shown in Figure $3 A$. Patients with IL/LL expression genotypes had significantly faster PWV than those with HL expression genotypes (7.93 \pm $1.38 \mathrm{~m} / \mathrm{s}$ versus $6.67 \pm 2.28 \mathrm{~m} / \mathrm{s}, p=0.027)$. Among the controls subjects, those having IL/LL expression genotypes also tended to have faster PWV than those with HL expression genotypes $(6.80 \pm 1.77 \mathrm{~m} / \mathrm{s}$ versus $5.86 \pm 1.30 \mathrm{~m} / \mathrm{s}, p=0.06)$.

Table 2. MBL genotype distribution in controls and the previously reported distribution in the Chinese population (ref. 30)

\begin{tabular}{lcc}
\hline Genotype & $\begin{array}{c}\text { Controls } \\
(n=41)\end{array}$ & $\begin{array}{c}\text { Population data } \\
(n=689)\end{array}$ \\
\hline$Y A / Y A$ & $16(39.0 \%)$ & $315(45.7 \%)$ \\
$Y A / X A$ & $11(26.8 \%)$ & $186(27.0 \%)$ \\
$Y A / O$ & $9(22.0 \%)$ & $116(16.8 \%)$ \\
$X A / O$ & $3(7.3 \%)$ & $35(5.1 \%)$ \\
$X A / X A$ & $1(2.4 \%)$ & $26(3.8 \%)$ \\
$O / O$ & $1(2.4 \%)$ & $11(1.6 \%)$ \\
\hline
\end{tabular}


Table 3. Distribution of MBL genotypes in patients and control subjects

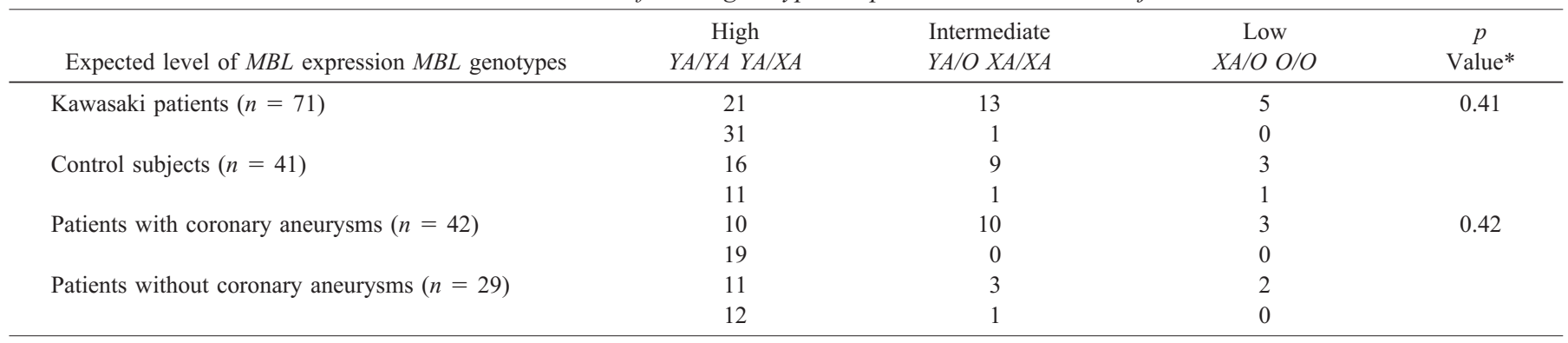

A $2 \times 2$ contingency table was created to compare the high $M B L$ expression categories $v s$ the combined intermediate and low $M B L$ expression categories.

* The $p$ values were calculated by Fisher's exact test.

We further determined whether $M B L$ genotype has differential effects on PWV in patients with and without coronary aneurysms (Fig. 3B). Among patients with coronary aneurysms, those with IL/LL expression genotypes had PWV faster, although not statistically significant, than those with HL expression genotypes $(7.50 \pm 1.41 \mathrm{~m} / \mathrm{s}$ versus $6.80 \pm 2.28 \mathrm{~m} / \mathrm{s}$, $p=0.32$ ). In contrast, among patients without coronary complications, those with IL/LL expression genotypes had significantly faster PWV than those with $\mathrm{HL}$ expression genotypes $(8.86 \pm 0.77 \mathrm{~m} / \mathrm{s}$ versus $6.48 \pm 2.32 \mathrm{~m} / \mathrm{s}, p=0.02)$.

Multiple linear regression analysis of the entire cohort of 112 subjects was used to identify significant determinants of PWV. The independent variables included were age at study, sex, body mass index, systolic and diastolic blood pressures, total cholesterol, patient status, and $M B L$ genotype subgroup. Significant determinants were age $(\beta=0.26, p=0.012)$, being a KD patient $(\beta=0.22, p=0.015)$, and $M B L$ IL/LL genotype subgroup $(\beta=0.20, p=0.03)$. When only the patient cohort was analyzed, with addition of duration since the onset of $\mathrm{KD}$ (age at study minus age at onset) into the multivariate model, only age $(\beta=0.26, p=0.043)$ and $M B L$ IL/LL genotype subgroup $(\beta=0.23, p=0.041)$ were found to be significant.

\section{DISCUSSION}

This study demonstrates significant modulating effects of $M B L$ genotypes on peripheral conduit arterial stiffness in pa-

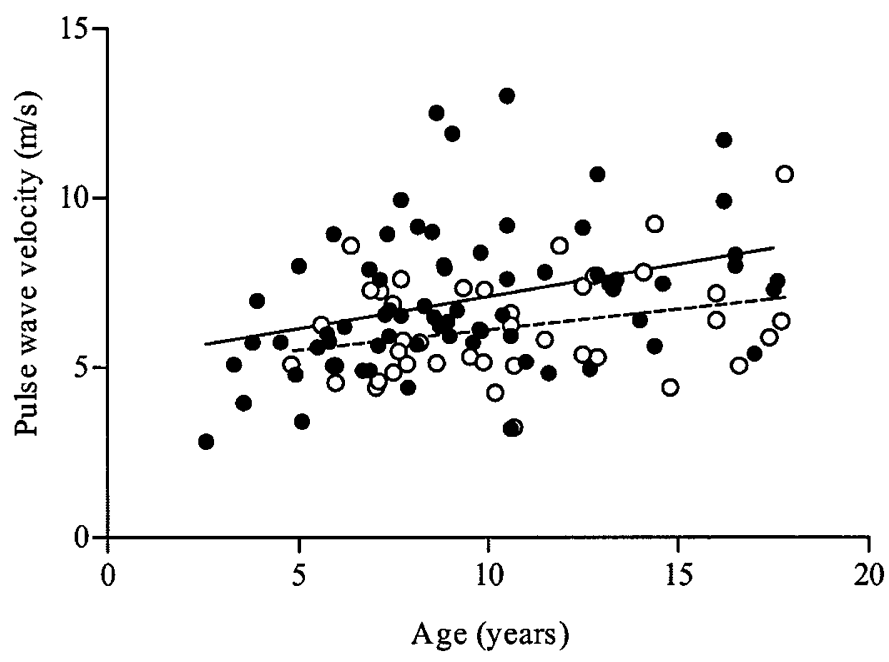

Figure 2. Linear regression of pulse wave velocity on age in patients (closed circles, solid line) and controls (open circles, dashed line). tients late after KD. The $M B L$ genotype influences arterial stiffness differently in patients with coronary aneurysms and those without coronary complications and controls. Patients with IL or LL $M B L$ expression genotypes have stiffer peripheral conduit arteries than those with HL $M B L$ expression genotypes. Furthermore, this genotype-modulating effect is more pronounced in patients without coronary aneurysms.

We (7) and the others (6) have previously reported on increased stiffness of the brachioradial arterial segment and carotid artery, respectively, in KD patients late after the acute illness. Importantly, the increase in stiffness is not limited to those with coronary complications (7). Although the exact mechanism remains unclear, replacement of elastic tissue by fibrous scar (31) and endothelial dysfunction (5) have been implicated. In this study, we further provide the first evidence to suggest that $M B L$ genotype may influence arterial stiffness after KD. Biezeveld et al. (17) have recently demonstrated an association between $M B L$ mutation and coronary artery lesions in Dutch patients with KD younger than $1 \mathrm{y}$ of age, but not in the older ones. However, we did not find significant differences in the genotype distributions between patients with and those without coronary aneurysms (Table 3 ). Whether this is related to ethnic difference awaits further clarification.

Whereas MBL has been considered as a modifier of susceptibility to infection (32), the notion that MBL may modulate disease progression in clinical diseases such as rheumatoid arthritis (33) and cystic fibrosis (29) is gaining support. The

Table 4. Comparisons of demographic and clinical data between patients with $H L \mathrm{MBL}$ expression genotypes and those with $I L / L L$ expression genotypes

\begin{tabular}{lccc}
\hline & $\begin{array}{c}\text { HL group } \\
(n=52)\end{array}$ & $\begin{array}{c}\text { IL/LL group } \\
(n=19)\end{array}$ & $p$ Value \\
\hline Age at study (y) & $9.5 \pm 3.7$ & $10.2 \pm 4.3$ & 0.31 \\
Median age (range) at & $1.5(0.3-10.8)$ & $0.9(0.3-10.3)$ & 0.32 \\
$\quad$ diagnosis of KD (y) & & & \\
Sex (male:female) & $34: 18$ & $14: 5$ & 0.58 \\
BMI (kg/m ${ }^{2}$ ) & $16.7 \pm 2.8$ & $16.7 \pm 2.7$ & 1.00 \\
IVIg given within first 10 d & $49: 3$ & $17: 2$ & 0.34 \\
$\quad$ of illness (yes:no) & & & \\
Coronary aneurysm (yes:no) & $29: 23$ & $13: 6$ & 0.42 \\
Follow-up duration (y) & $7.5 \pm 3.8$ & $8.3 \pm 4.1$ & 0.44 \\
Total cholesterol (mmol/L) & $4.4 \pm 0.8$ & $4.4 \pm 0.9$ & 0.67 \\
SBP (mm Hg) & $107 \pm 12$ & $112 \pm 12$ & 0.12 \\
DBP (mm Hg) & $58 \pm 7$ & $58 \pm 11$ & 0.86 \\
\hline
\end{tabular}

BMI, body mass index; DBP, diastolic blood pressure; SBP, systolic blood pressure. 

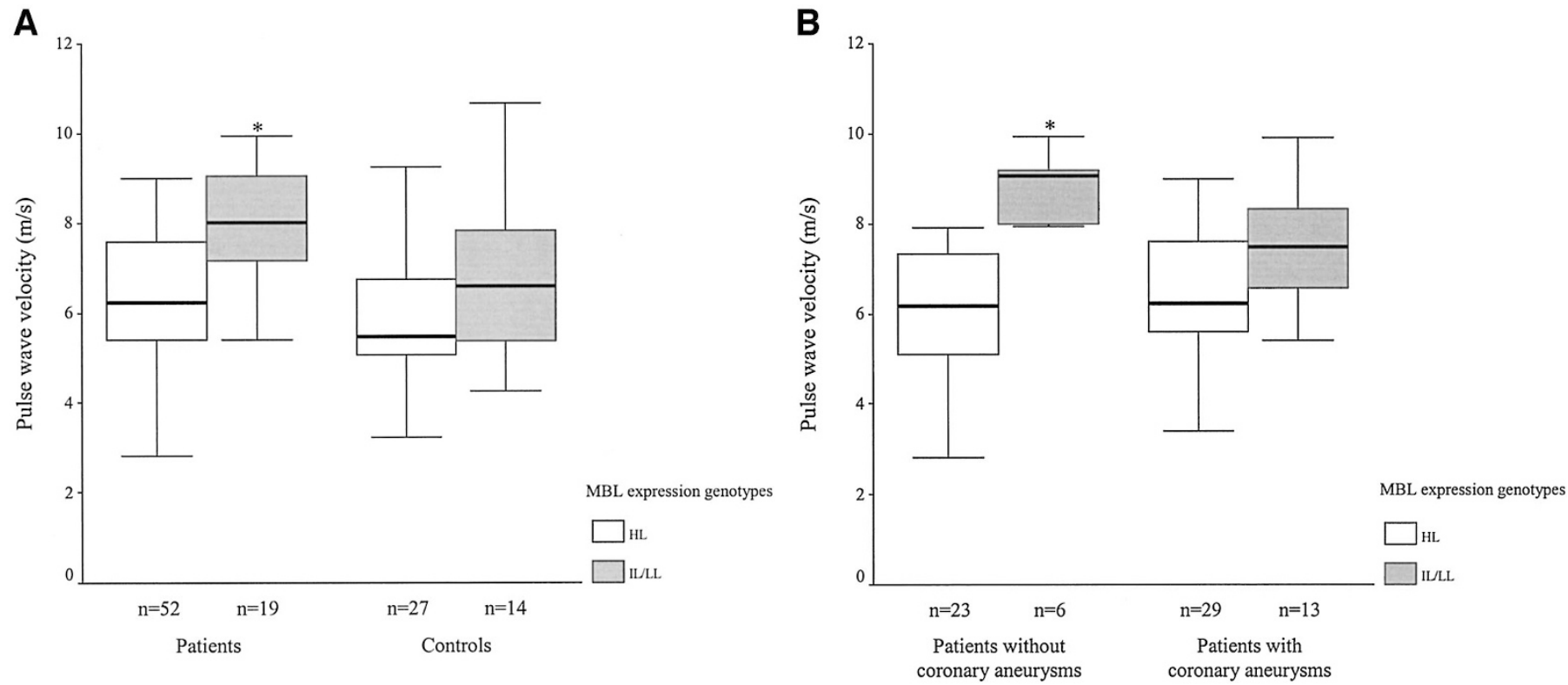

Figure 3. Cluster box plots of PWV by $M B L$ expression genotypes in $(A)$ patients and controls $\left({ }^{*} p=0.027\right.$ vs patients with HL expression genotypes), and $(B)$ patients with and without coronary aneurysms $\left({ }^{*} p=0.02\right.$ s patients without coronary aneurysms but having HL $M B L$ expression genotypes).

recently reported associations between MBL deficiency and cardiovascular abnormalities are intriguing. Madsen et al. (18) reported on the association of MBL deficiency with severe atherosclerosis and speculated that the mechanism may be related to modulation of the severity of Chlamydia pneumoniae infection by MBL. Indeed, Rugonfalvi-Kiss et al. (34) has subsequently shown that infection with $C$. pneumoniae leads to development and progression of severe coronary artery disease mainly in patients with variation in the $M B L$ gene. The findings of the present study further suggest that MBL may impact on cardiovascular morbidities through modulation of systemic arterial stiffness after vasculitic damage.

The mechanism by which $M B L$ genotype modulates arterial stiffness in patients with KD remains speculative. Although the etiology of KD is still elusive, epidemiologic and clinical data strongly suggest that an infectious agent is probably the inciting factor (35). A low serum MBL level may perhaps be associated with less effective opsonization and delayed clearance of the triggering infectious agent, resulting in more significant arterial inflammation in the acute phase and dysfunction in the long term. Additionally, the low MBL level may result in less effective clearance of circulating immune complexes, present during the subacute and convalescent phases of KD (36), as a result of impaired complement fixation. Although the pathogenic role of circulating immune complexes in KD remains uncertain, a higher level has been reported in patients with than those without coronary complications (37). Furthermore, MBL may modulate severity of inflammation through a dose-dependent influence on proinflammatory cytokine production (38). Interestingly, in an experimental model of postischemic myocardial reperfusion injury, Jordan et al. (39) demonstrated a proinflammatory and possibly a pathophysiological role of MBL-mediated complement activation. Hence, low and high levels of MBL may perhaps both play a role in different inflammatory pathophysiologies, probably through interactions with other factors and pathways.

The more pronounced modulating effect of $M B L$ genotype on arterial stiffness in patients without coronary artery aneurysms is even more intriguing. As the severity of vasculitis in the acute phase is, to some extent, reflected by development of coronary lesions (40), our findings suggest that the modulating effects of $M B L$ genotype depends on severity of vascular inflammation. Indeed, the possibility of genetic programming of the dynamic response to inflammatory stimuli rather than the relatively static baseline serum MBL levels may well underlie the modulating influence of $M B L$ genotypes. Thus, whereas the modulating effects of $M B L$ genotype on arterial stiffness tend almost to be significant in controls, superimposed mild-tomoderate degree of vascular inflammation as a result of KD unmasks the potentially protective role of MBL on long-term arterial function. However, this potentially protective effect of MBL might be overwhelmed by severe inflammation.

A number of limitations to this study deserve comment. First, it would have been ideal to categorize the subjects into three MBL genotype subgroups for analysis. The limited number of subjects with LL expression genotypes, however, would have limited the statistical power to identify genuine differences between groups. Nonetheless, the expected genotype distribution in our controls is an assurance of an unbiased sample. Second, the proportion of our patients with coronary aneurysms $(59 \%, 42 / 71)$ is higher than that expected after i.v. Ig therapy. This is likely to be related to tertiary referral bias. Nonetheless, this provides an opportunity to stratify the patients based on coronary complications for determination of possible differential effects of $M B L$ genotype on arterial stiffness in these subgroups.

In conclusion, this study demonstrates that $M B L$ genotype influences arterial stiffness, an important cardiovascular risk 
factor, in children after KD. Determination of the $M B L$ genotype status in KD patients, regardless of coronary artery complications, may help to identify those potentially at risk of premature atherosclerosis for long-term follow-up.

\section{REFERENCES}

1. Taubert KA, Rowley AH, Shulman ST 1994 Seven-year national survey of Kawasaki disease and acute rheumatic fever. Pediatr Infect Dis J 13:704-708

2. Kato H, Sugimura T, Akagi T, Sato N, Hashino K, Maeno Y, Kazue T, Eto G, Yamakawa R 1996 Long-term consequences of Kawasaki disease. A 10- to 21-year follow-up study of 594 patients. Circulation 94:1379-1385

3. Sugimura T, Kato H, Inoue O, Takagi J, Fukuda T, Sato N 1992 Vasodilatory response of the coronary arteries after Kawasaki disease: evaluation by intracoronary injection of isosorbide dinitrate. J Pediatr 121:684-688

4. Iemura M, Ishii M, Sugimura T, Akagi T, Kato H 2000 Long term consequences of regressed coronary aneurysms after Kawasaki disease: vascular wall morphology and function. Heart 83:307-311

5. Dhillon R, Clarkson P, Donald AE, Powe AJ, Nash M, Novelli V, Dillon MJ, Deanfield JE 1996 Endothelial dysfunction late after Kawasaki disease. Circulation 94:2103-2106

6. Noto N, Okada T, Yamasuge M, Taniguchi K, Karasawa K, Ayusawa M, Sumitomo N, Okada T, Harada K 2001 Noninvasive assessment of the early progression of atherosclerosis in adolescents with Kawasaki disease and coronary artery lesions. Pediatrics 107:1095-1099

7. Cheung YF, Yung TC, Tam SC, Ho MH, Chau AK 2004 Novel and traditional cardiovascular risk factors in children after Kawasaki disease: implications for premature atherosclerosis. J Am Coll Cardiol 43:120-124

8. Newburger JW, Burns JC, Beiser AS, Loscalzo J 1991 Altered lipid profile after Kawasaki syndrome. Circulation 84:625-631

9. Pilla CB, Cheung YF, Brogan PA, Dillon MJ, Redington AN 2000 Chronically reduced arterial distensibility in Kawasaki disease: further evidence for the beneficial effects of immunoglobulin. Circulation 102(suppl II):830

10. Turner MW 1996 Mannose-binding lectin: the pluripotent molecule of the innate immune system. Immunol Today 17:532-540

11. Matsushita M, Fujita T 1992 Activation of the classical complement pathway by mannose-binding protein in association with a novel C1s-like serine protease. J Exp Med 176:1497-1502

12. Thiel S, Vorup-Jensen T, Stover CM, Schwaeble W, Laursen SB, Poulsen K, Willis AC, Eggleton P, Hansen S, Holmskov U, Reid KB, Jensenius JC 1997 A second serine protease associated with mannan-binding lectin that activates complement. Nature 386:506-510

13. Sumiya M, Super M, Tabona P, Levinsky RJ, Arai T, Turner MW, Summerfield JA 1991 Molecular basis of opsonic defect in immunodeficient children. Lancet 337:1569-1570

14. Garred P, Madsen HO, Hoffmann B, Svejgaard A 1995 Increased frequency of homozygosity of abnormal mannan-binding-protein alleles in patients with suspected immunodeficiency. Lancet 346:941-943

15. Summerfield JA, Sumiya M, Levin M, Turner MW 1997 Association of mutations in mannose binding protein gene with childhood infection in consecutive hospital series. BMJ 314:1229-1232

16. Summerfield JA, Ryder S, Sumiya M, Thursz M, Gorchein A, Monteil MA, Turne MW 1995 Mannose binding protein gene mutations associated with unusual and severe infections in adults. Lancet 345:886-889

17. Biezeveld MH, Kuipers IM, Geissler J, Lam J, Ottenkamp JJ, Hack CE, Kuijpers TW 2003 Association of mannose-binding lectin genotype with cardiovascular abnormalities in Kawasaki disease. Lancet 361:1268-1270

18. Madsen HO, Videm V, Svejgaard A, Svennevig JL, Garred P 1998 Association of mannose-binding-lectin deficiency with severe atherosclerosis. Lancet 352:959-960
19. Kato H, Inoue O, Kawasaki T, Kawasaki T, Fujiwara H, Watanabe T, Toshima H 1992 Adult coronary artery disease probably due to childhood Kawasaki disease. Lancet 340:1127-1129

20. Burns JC, Shike H, Gordon JB, Halhotra A, Schoenwetter M, Kawasaki T 1996 Sequelae of Kawasaki disease in adolescents and young adults. J Am Coll Cardiol 28:253-257

21. Lipscombe RJ, Beatty DW, Ganczakowski M, Goddard EA, Jenkins T, Lau YL, Spurdle AB, Sumiya M, Summerfield JA, Turner MW 1996 Mutation in the human mannose-binding protein gene: frequencies in several population groups. Eur J Hum Genet 4:13-19

22. Madsen HO, Garred P, Kurtzhals JA, Lamm LU, Ryder LP, Thiel S, Svejgaard A 1994 A new frequent allele is the missing link in the structural polymorphism of the human mannan-binding protein. Immunogenetics 40:37-44

23. Madsen HO, Garred P, Thiel S, Kurtzhals JA, Lamm LU, Ryder LP, Svejgaard A 1995 Interplay between promoter and structural gene variants control basal serum level of mannan-binding protein. J Immunol 155:3013-3020

24. Fujiwara H, Hamashima Y 1978 Pathology of the heart in Kawasaki disease Pediatrics 61:100-107

25. Nichols WW, O'Rourke MF 1998 Vascular impedance. In: McDonald's Blood Flow in Arteries: Theoretical, Experimental and Clinical Principles. Edward Arnold, London, UK, pp 54-97, 243-293

26. Loukogeorgakis S, Dawson R, Phillips N, Martyn CN, Greenwald SE 2002 Validation of a device to measure arterial pulse wave velocity by a photoplethysmographic method. Physiol Meas 23:581-596

27. Lau YL, Chan SY, Turner MW, Fong J, Karlberg J 1995 Mannose-binding protein in preterm infants: developmental profile and clinical significance. Clin Exp Immunol 102:649-654

28. Garred P, Madsen HO, Svejgaard A 1996 Genetics of human mannan-binding protein. In: Ezekowitz RAB, Sastry K, Reid KBM (eds) Collectins and Innate Immunity. RG Landes, Austin, TX, pp 139-164

29. Garred P, Presseler T, Madsen HO, Frederiksen B, Svejgaard A, Hoiby N, Schwartz M, Koch C 1999 Association of mannose-binding lectin gene heterogeneity with severity of lung disease and survival in cystic fibrosis. J Clin Invest 104:431-437

30. Ip WK, To YF, Cheng SK, Lau YL 2004 Serum mannose-binding lectin levels and mbl2 gene polymorphisms in different age and gender groups of southern Chinese adults. Scand J Immunol 59:310-314

31. Amano S, Hazama F, Hamashima Y 1979 Pathology of Kawasaki disease: I Pathology and morphogenesis of the vascular changes. Jpn Circ J 43:633-643

32. Neth O, Hann I, Turner MW, Klein NJ 2001 Deficiency of mannose-binding lectin and burden of infection in children with malignancy: a prospective study. Lancet 358:614-618

33. Ip WK, Lau YL, Chan SY, Mok CC, Chan D, Tong KK, Lau CS 2000 Mannosebinding lectin and rheumatoid arthritis in southern Chinese. Arthritis Rheum 43:1679-1687

34. Rugonfalvi-Kiss S, Endresz V, Madsen HO, Burian K, Duba J, Prohaszka Z, Karadi I, Romics L, Gonczol E, Fust G, Garred P 2002 Association of Chlamydia pneumoniae with coronary artery disease and its progression is dependent on the modifying effect of mannose-binding lectin. Circulation 106:1071-1076

35. Rowley AH, Shulman ST 1999 Kawasaki syndrome. Pediatr Clin North Am 46:313329

36. Salo E, Kekomaki R, Pelkonen P, Ruuskanen O, Viander M, Wagner O 1988 Kawasaki disease: monitoring of circulating immune complexes. Eur J Pediatr 147:377-380

37. Ono S, Onimaru T, Kawakami K, Hokonohara M, Miyata K 1985 Impaired granulocyte chemotaxis and increased circulating immune complexes in Kawasaki disease. J Pediatr 106:567-570

38. Jack DL, Klien NJ, Turner MW 2001 Mannose-binding lectin: targeting the microbial world for complement attack and opsonophagocytosis. Immunol Rev 180:86-99

39. Jordan JE, Montalto MC, Stahl GL 2001 Inhibition of mannose-binding lectin reduces postischemic myocardial reperfusion injury. Circulation 104:1413-1418

40. Koren G, Lavi S, Rose V, Rowe R 1986 Kawasaki disease: review of risk factors for coronary aneurysms. J Pediatr 108:388-392 